\title{
Alternative Thoracic Drainage Strategy Alleviates Perioperative Pain After Lung Wedge Resection: A Randomized Controlled Trial
}

\section{Shoucun Wei}

Guangdong Provincial People's Hospital

\section{Guangyan Zhang}

Guangdong Provincial People's Hospital

Jue Ma

Guangdong Provincial People's Hospital

\section{Lidan Nong}

Guangdong Provincial People's Hospital

Jiatao Zhang

Guangdong Lung Cancer Institute

Wenzhao Zhong

Guangdong Lung Cancer Institute

Jianxiu Cui ( $\nabla$ cuijianxiu@163.com )

Guangdong Provincial People's Hospital

\section{Research Article}

Keywords: thoracic drainage tube, double lumen central venous catheter, video-assisted thoracic surgery, acute pain, chronic pain

Posted Date: January 20th, 2021

DOl: https://doi.org/10.21203/rs.3.rs-142647/v1

License: (c) (1) This work is licensed under a Creative Commons Attribution 4.0 International License. Read Full License 
Title: Alternative thoracic drainage strategy alleviates perioperative pain after lung wedge resection: a randomized controlled trial

Shoucun Wei ${ }^{1,3}$, Guangyan Zhang ${ }^{1}$, Jue MA ${ }^{1}$, Lidan Nong ${ }^{1}$, Jiatao Zhang ${ }^{2}$, Wenzhao Zhong $^{2}$, Jianxiu Cui ${ }^{1}$

${ }^{1}$ Department of Anesthesiology, Guangdong General Hospital, Guangzhou, Guangdong, China.

${ }^{2}$ Guangdong Lung Cancer Institute, Guangzhou, Guangdong, China.

${ }^{3}$ Shantou University Medical College, Shantou, Guangdong, China

Corresponding author: Jianxiu Cui

Tel: 02083827812

E-mail: cuijianxiu@163.com

Institutional Address: No. 106 Zhongshan Er Road, Yuexiu Area, Guangzhou, Guangdong Province, P.R. China. 


\section{Alternative thoracic drainage strategy alleviates perioperative pain after lung wedge resection: a randomized controlled trial}

\section{Abstract \\ Background}

Indwelling thoracic drainage tube is one of the leading causes of postoperative pain after thoracotomy. Currently, limited evidence exists on evaluating the acute and chronic pain resulting from chest tube after video-assisted thoracic surgery (VATS). Herein, we intended to explore an alternative drainage method and its impact on acute and chronic pain after VATS.

\section{Methods}

For this purpose, ninety-two patients undergoing lung wedge resection were selected and randomly assigned to conventional chest tube (CT) group and venous catheter (VC) group, respectively. Then, the numeric rating scale (NRS) and pain DETECT questionnaire were applied to evaluate the level and characteristics of postoperative pain.

\section{Results}

Our data showed that the NRS scores of the VC group during hospitalization were significantly lower than those of the CT group 6 hours after surgery, at postoperative day 1 , at postoperative day 2 , and at the moment of drainage tube removal), respectively. Moreover, the number of postoperative salvage analgesics such as nonsteroidal anti-inflammatory drugs (NSAIDs) used in the VC group and postoperative hospitalization days were notably reduced compared with the CT group. However, no significant difference was observed in terms of NRS pain scores between 
the two groups of patients during the follow-up for chronic pain at 3 months and 6 months.

\section{Conclusion}

Taken together, the drainage strategy mentioned in this article is effective in relieving perioperative acute pain and reducing the use of salvage analgesics in selected patients undergoing VATS wedge resection, which is beneficial for the rapid recovery of patients after surgery.

\section{Trial registration}

ClinicalTrials.gov, NCT03230019. Registered July 23, 2017

Keywords: thoracic drainage tube, double lumen central venous catheter, video-assisted thoracic surgery, acute pain, chronic pain 


\section{Background}

Favorable pain management after thoracotomy is of great significance to avoid postoperative chronic pain and complications ${ }^{[1-3]}$. Interestingly, evidence exists reporting that the incidence of acute pain is $59-90 \%{ }^{[4-6]}$, and $11-35 \%$ of the patients develop to chronic pain ${ }^{[7-11]}$ after surgery through video-assisted thoracoscopic surgery (VATS), which inflicts enormous burden on the postoperative rehabilitation of patients. It has been well recognized that postoperative pain is mainly caused by surgical trauma, intercostal nerve injury, indwelling thoracic drainage tube, and social psychological factors and so on ${ }^{[12-14]}$. Increasing studies have reported the treatment outcomes of different surgical approaches (thoracotomy or thoracoscopic surgery) and perioperative peripheral nerve injury on postoperative pain of patients ${ }^{[4]}$. However, limited clinical investigations focus on evaluating the impact of thoracic drainage tube on postoperative pain of VATS.

In recent years, with the popularization of enhanced recovery after surgery (ERAS), the tubeless strategy ${ }^{[15-16]}$ is increasingly favored by surgeons and patients. Thus, seeking safe alternative for conventional chest tube or even abolishing the drainage tube has become a mainstream trend for many surgeons in minimally invasive pulmonary surgery. In our previous reported study, we provided evidence demonstrating that the alternative of conventional chest drainage by 7-Fr double-lumen central venous catheter ${ }^{[17]}(\mathrm{CVC})$ along with a prophylactic air-extraction strategy ${ }^{[18]}$ can be safely applied to patients under lung wedge resection through VATS without causing much postoperative complications.

Intriguingly, we speculated that the alternative of conventional chest drainage strategy by 7-Fr double-lumen CVC may reduce the postoperative pain after lung wedge 
resection through VATS in the present study. To verify this hypothesis, we designed a single-center, prospective, and randomized controlled trial to explore the effects of different drainage methods on acute and chronic pain of patients after lung wedge resection under VATS.

\section{Materials and Methods}

\section{Ethical statement and Registration}

This prospective, single-center, open-label, and randomized controlled trial was approved by the Ethics Committee of Guangdong General Hospital (No. GDREC 2017261H) and registered before patient enrollment at www.clinicaltrials.gov on July 23, 2017 (registration number: NCT03230019). All participants signed written informed consents before enrollment in the study.

\section{Inclusion and exclusion criteria}

The subjects for the screening mainly include patients who received lung wedge resection through VATS at the Guangdong General Hospital and their age should be above 18 years old. The preoperative exclusion criteria were as follows: (1) any unstable systemic diseases such as active infection, poorly controlled hypertension within 3 months, diabetes or unstable angina; (2) history of ipsilateral chest surgery; (3) preoperative chest X-ray showing pneumonia or atelectasis; (4) bleeding tendency; (5) administration of anticoagulant drugs; or (6) history of other chronic chest pain. Patients were excluded if they needed to receive segmentectomy or lobectomy, or if they showed severe adhesions during the surgery, requiring further exploratory surgery, or if air leaks were detected during leak examinations (Figure 2).

\section{Sample size}


This present study aimed to validate that the drainage strategy of the VC group after lung wedge resection through VATS could ameliorate the postoperative pain. A previous retrospective study ${ }^{[18]}$ has documented that the mean values of NRS scores of postoperative acute pain in the conventional CT group and VC group were 3.4 and 2.3, while standard deviations were 1.1 and 0.8 , respectively. The bilateral $\alpha$ was 0.01 , and the power was $95 \%$, in which half of the participants were assigned into the CT group and the rest into the VC group. The loss of follow-up and refusal to follow-up was calculated as $20 \%$. Moreover, thirty-eight patients were included in the CT group and VC group, respectively, which was quantified by PASS software (version 15.0; NCSS, Kaysville, UT, USA). Hence, the total number of participants was at least 76.

\section{Randomization and blinding}

After surgical incision (before wedge resection), one surgical team member randomly generated codes by using simple random sampling and SAS statistical software (SAS Institute, Cary, NC, USA) to randomly assign patients to the $\mathrm{VC}$ group or conventional CT group in a 1:1 ratio. In this study, the VC group didn't carry a chest drainage bottle after surgery, which was easy to be identified by the patient and the medical staff, thus the blind method was not designed herein.

\section{Anesthesia and surgery}

All patients received surgeries after bronchial intubation under general anesthesia. The anesthesia induction and management were performed by the same group of anesthesiologists. After surgery, patient was sent to post-anesthesia care unit (PACU), received uniformly formulated intravenous analgesia containing opioids (sufentanil 2 $\mu \mathrm{g} / \mathrm{h}$ ) with a disposable balloon infuser (Accufuser). An appropriate amount of rescue analgesics was administrated according to the pain level after returning to the ward. The 
salvage analgesic measures referred to that patients with mild pain were given nonsteroidal anti-inflammatory drugs (NSAIDs) (such as Celebrex 200 mg, Flurbiprofen Axetil injection $50 \mathrm{mg}$ ), patients with moderate pain were treated with tramadol (50-100 mg), and patients with severe pain were treated with morphine (7-10 $\mathrm{mg}$ ). Before skin incision, $2 \%$ lidocaine was used for local infiltration anesthesia along the incision. A three-centimeter long incision was made between the anterior axillary line and the fourth or fifth intercostal line of the axillary line, followed by the placement of an incision protective sleeve. However, participants were excluded from the study in the case of severe adhesions or necessity of lobectomy due to insufficient surgical margin during exploratory process. They were subjected to CT drainage, and all wedge resections were performed using a linear cutting stapler (Ethicon, Cincinnati, USA or Medtronic, Minneapolis, USA). After the wedge resection, the specified drainage strategy was carried out. In the VC group, a double-lumen CVC $(7-\mathrm{Fr} \times 20 \mathrm{~cm})$ was inserted into the second intercostal space using a puncture kit. After the closure of the incision, a $50 \mathrm{ml}$ syringe was used to extract air through the catheter for air leak test to avoid obvious air leakage ${ }^{[18]}$. The patients in the CT group were inserted with 20-Fr chest tube for drainage (Fig 1). After the incision was closed, the chest tube was immersed in water and the patient lung was gradually inflated to conduct an air-leak test to ensure that there was no obvious air leakage. However, patients with air leakage during the test were excluded from the study and received CT drainage. All the patients underwent a chest X-ray examination on the first day after surgery. Upon the observation of a large amount of pneumothorax, the CT group may be required to strengthen deep breathing and coughing exercises or for 8-10-cm H20 suction via the chest tube. In the scenario of massive pneumothoraxes in the VC group, a syringe was 
applied to perform a prophylactic air-extraction strategy for about 3 times a day through the available catheter or reinserting chest tube. The chest tube/catheter extraction was considered upon indications of blood oxygen saturation $(\geq) 95 \%$, fully dilated lungs, and no air leakage in the CT group.

\section{Pain evaluation}

The chronic pain was defined as postoperative pain lasting longer than 3 months according to the International Association for the Study of Pain ${ }^{[11]}$ and a numeric rating scale (NRS) was used to assess the postoperative pain level of patients. A score of 0 indicated no pain while a score of 10 represented the worst pain. On the basis of the evaluation results, the pain level was divided into 4 grades: painless $(\mathrm{NRS}=0)$, mild (NRS 1-3), moderate (NRS 4-6), and severe (NRS 7-10). During the preoperative visit, the anesthesiologist showed the patient how to use the NRS for the pain assessment. The patient's pain assessment was conducted in two stages, including the perioperative period and the assessment of chronic pain at 3 and 6 months after surgery. The perioperative evaluation referred to the evaluation during the hospitalization and 1 month after the operation. A total of 6 pain evaluations were performed during the hospitalization, which was the first 6 hours after patients returned to the ward, 8 am and $6 \mathrm{pm}$ on the first day after the surgery, 8 am and $6 \mathrm{pm}$ on the second day after surgery, at the time of extubation and after the doctor issued a discharge from the hospital. In the follow-up of chronic pain, the pain DETECT questionnaire (PD-Q) was used to investigate the characteristics of the pain ${ }^{[19]}$, which included allodynia, insufficiency, hyperalgesia, numbness, tingling, burning pain, and soreness.

\section{Study endpoints}


The major endpoint of this study was the pain level of acute pain after surgery on the first day. The secondary observation indicators mainly included the duration of postoperative intravenous analgesia use, the number of postoperative salvage analgesics during acute pain attacks, the level of pain during extubation, the duration of postoperative drainage, postoperative hospitalization days, the state of the pain 1 month after surgery, and the level and characteristics of chronic pain of patients 3 and 6 months after surgery.

\section{Statistical analysis}

The quantitative data were presented as the mean \pm standard deviation (mean $\pm \mathrm{SD}$ ).

The normally distributed data between two groups were compared using unpaired $t$-test, and the data with skewed distribution were compared using the Mann-Whitney U test. Moreover, count data were presented by actual number of cases and percentages, which were processed by the Chi-square and Fisher's exact test. All the data were analyzed by SPSS 25 software (IBM, Armonk, NY, USA), with $P<0.05$ as a level of statistically significance.

\section{Results}

From August 2017 to October 2018, a total of 102 patients with proposed wedge resection met the inclusion criteria for this study. Among them, 94 patients signed written informed consents, and the surgeries of 2 patients were temporarily canceled, so 92 patients were randomly assigned to $\mathrm{CT}$ group and VC group. Besides, the cases with intraoperative surgery type change, air leakage, and severe adhesion were excluded. Finally, 42 patients were included in the CT group and 38 patients were assigned in the VC group (Fig 2). Our results showed that the demographics, classification of American 
Society of Anesthesiologists (ASA), and surgical characteristics of the patients of two groups were comparable, and no inpatient deaths or intensive care cases were identified (Table 1).

\section{Outcomes}

The obtained data suggested that the NRS scores of the VC group during hospitalization were considerably lower than those of in the CT group at 6 hours after surgery $(2.3 \pm$ 0.9 vs. $2.9 \pm 0.8, P=0.001)$, postoperative day $1(2.6 \pm 0.9$ vs. $2.8 \pm 0.8, P=0.026)$, postoperative day $2(2.2 \pm 0.8 v s .2 .7 \pm 0.7, P=0.009)$, and CT removal $(2.8 \pm 0.7 v s$. $3.3 \pm 0.7, P=0.003)$, respectively. Moreover, no statistically significant difference was observed regarding the pain level of two groups of patients on discharge $(1.3 \pm 0.7 v s$. $1.5 \pm 0.6, P=0.267)($ Table 2$)$.

Furthermore, the findings demonstrated that the number of postoperative salvage analgesics such as NSAIDs used $(2.0 \pm 1.5$ vs. $3.0 \pm 2.0, P=0.023)$ and postoperative hospitalization days $(2.7 \pm 1.4 v s .3 .2 \pm 1.2, P=0.001)$ were remarkably reduced in the VC group, when compared with the CT group. However, the difference didn't differ significantly in terms of pain level and characteristics between the two groups, including the number of postoperative salvage opioid use $(P=0.545)$, days of postoperative venous analgesia $(1.1 \pm 0.4 v s .1 .3 \pm 0.6, P=0.069)$, and pain level at 1 month $(0.9 \pm 0.5$ vs. $1.1 \pm 0.5, P=0.182), 3$ months $(P=0.783)$ and 6 months $(P=$ 0.521) after surgery, respectively (Table 2-3). In the chronic pain follow-up, both groups of patients had different degrees of abnormal feelings, which were manifested as sensory changes related to weather change, mainly including paresthesia, numbness and tingling (Table 3). The degree of discomfort did not affect sleep and daily activities, and no patient needed long-term pain medication. 


\section{Discussion}

Thoracotomy has been reported to cause severe pain and trauma, while surgical trauma has been considerably reduced due to the development of VATS ${ }^{[20]}$. Moreover, with the popularization of ERAS, more precise management is required for the perioperative period. In addition to minimally invasive surgery and multi-modal analgesia, optimizing the management of various tubes, or even abolishing urethral catheter, postoperative drainage tube in certain types of surgery could be of great value in reducing the discomfort of the patients.

In the present trial, we compared the effects of drainage tubes with different diameters on postoperative pain, indirectly proved that chest tube is one of the causes of postoperative acute pain, and smaller diameter thoracic drainage tube had a significant effect on relieving perioperative pain. Here we preferred using 7-Fr central venous catheter as the drainage tube after wedge resection of lung, because it is small in size compared with routine chest tube, hardly prone to air leakage, but still could ensure a certain gas drainage efficiency. Moreover, indwelling this catheter causes slighter stimulation to pleura, which is beneficial for wound healing ${ }^{[21]}$ and can effectively relieve the pain caused by indwelling chest tube after operation. Thus, it is a safe and effective alternative drainage strategy before we truly achieve tube-free, especially suitable for patients who estimated with limited postoperative exudate.

With regard to chronic pain, our research results only suggested that there was no difference in the effects of the two kinds of pipes on abnormal skin sensation within half a year after operation. It is hard to conclude that thoracic drainage tube has nothing to do with postoperative chronic pain. We need more conclusive evidence to explain the connection between chest tube and postoperative chronic pain. 
Pneumothorax occurred in 4 patients in VC group, but all patients recovered well after salvage air extraction and did not need to reinsert the chest tube. There is no difference in postoperative complications between the two groups.

We acknowledge that the trial has its limitations and that restrictive inclusion criteria may impede the universality and applicability of the results. First of all, the diameter of the catheter is small, the drainage capacity of pleural effusion is limited, and it is easy to be blocked. In order to avoid pneumothorax and massive pleural effusion after operation, it is very dependent on postoperative X-ray examination and dynamic observation of patients' reactions, especially at the beginning of the study. Secondly, our study focused on the data and observations of a single center of research and the sample size was relatively limited. Last, in order to directly explain the contribution of chest tube to postoperative acute and chronic pain, it is necessary to further explore the difference between the patients without chest tube and the control group in the followup study.

\title{
Conclusion
}

Taken together, the drainage strategy mentioned in this article is effective in relieving perioperative acute pain and reducing the use of salvage analgesics in selected patients undergoing VATS wedge resection, which is beneficial for the rapid recovery of patients after surgery.

\author{
Abbreviations \\ VATS: Video-assisted Thoracic Surgery \\ NRS: Numeric Rating Scale
}


BMI: Body Mass Index

ASA: American Society of Anesthesiologists

PACU: Post-anesthesia Care Unit

NSAIDs: Nonsteroidal Antiinflammatory Drugs

ERAS: Enhanced Recovery after Surgery

BIS: Bispectral Index

\section{Acknowledgements}

Not applicable.

\section{Authors' contributions}

JXC and SCW contributed to the conception and design of the work. JTZ and WZZ performed patient recruitment, randomization, and allocation. NLD and JM performed anesthesia. GYZ analyzed data, prepared figures and tables. SCW and JXC wrote the manuscript. All authors revised the manuscript and approved the final version to be published.

\section{Funding}

None

\section{Availability of data and materials}

The datasets used and/or analyzed during the current study are available from the corresponding author on reasonable request.

\section{Ethics approval and consent to participate}


The study was approved by the Ethics Committee of Guangdong General Hospital (No. GDREC 2017261H) and registered on July 23, 2017 (No. NCT03230019). It strictly abided by the Declaration of Helsinki and CONSORT Standards.All participants signed written informed consents before enrollment in the study.

\section{Consent for publication}

Not applicable.

\section{Competing interests}

The authors declare that they have no competing interests

\section{Author details}

${ }^{1}$ Department of Anesthesiology, Guangdong General Hospital, Guangzhou, Guangdong, China. ${ }^{2}$ Guangdong Lung Cancer Institute, Guangzhou, Guangdong, China. ${ }^{3}$ Shantou University Medical College, Shantou, Guangdong, China

\section{References:}

1. Watanabe S, Imai K, Kimura T, Saito Y, Takashima S, Matsuzaki I, et al. Effect of lidocaine cream analgesia for chest drain tube removal after video-assisted thoracoscopic surgery for lung cancer: a randomized clinical trial. Regional Anesthesia \& Pain Medicine. 2019; 45:16-21.

2. Kehlet H, Jensen TS, Woolf CJ. Persistent postsurgical pain: risk factors and prevention. The Lancet. 2006; 367:1618-1625. 
3. Katz J, Jackson M, Kavanagh BP, Sandler AN. Acute Pain after Thoracic Surgery Predicts Long-Term Post-Thoracotomy Pain. The Clinical Journal of Pain. 1996; 12:50-55.

4. Takenaka S, Saeki A, Sukenaga N, Ueki R, Kariya N, Tatara T, et al. Acute and chronic neuropathic pain profiles after video-assisted thoracic surgery. MEDICINE. 2020;99: e19629.

5. Bayman EO, Parekh KR, Keech J, Larson N, Vander WM, Brennan TJ. Preoperative Patient Expectations of Postoperative Pain Are Associated with Moderate to Severe Acute Pain After VATS. PAIN MED. 2019; 20:543-554.

6. Wang H, Li S, Liang N, Liu W, Liu H, Liu H. Postoperative pain experiences in Chinese adult patients after thoracotomy and video-assisted thoracic surgery. $J$ CLIN NURS. 2017; 26:2744-2754.

7. Wildgaard K, Ringsted TK, Hansen HJ, Petersen RH, Kehlet H. Persistent postsurgical pain after video-assisted thoracic surgery - an observational study. ACTA ANAESTH SCAND. 2016; 60:650-658.

8. WILDGAARD K, RAVN J, NIKOLAJSEN L, JAKOBSEN E, JENSEN TS, KEHLET H. Consequences of persistent pain after lung cancer surgery: a nationwide questionnaire study. ACTA ANAESTH SCAND. 2011; 55:60-68.

9. Shanthanna H, Aboutouk D, Poon E, Cheng J, Finley C, Paul J, et al. A retrospective study of open thoracotomies versus thoracoscopic surgeries for persistent postthoracotomy pain. J CLIN ANESTH. 2016; 35:215-220.

10. Kwon ST, Zhao L, Reddy RM, Chang AC, Orringer MB, Brummett CM, et al. Evaluation of acute and chronic pain outcomes after robotic, video-assisted 
thoracoscopic surgery, or open anatomic pulmonary resection. The Journal of Thoracic and Cardiovascular Surgery. 2017; 154:652-659.

11. Bayman EO, Parekh KR, Keech J, Selte A, Brennan TJ. A Prospective Study of Chronic Pain after Thoracic Surgery. ANESTHESIOLOGY. 2017; 126:938-951.

12. Perkins FM, Kehlet H. Chronic pain as an outcome of surgery. A review of predictive factors. ANESTHESIOLOGY. 2000; 93:1123-1133.

13. Kaplowitz J, Papadakos PJ. Acute Pain Management for Video-Assisted Thoracoscopic Surgery: An Update. J CARDIOTHOR VASC AN. 2012; 26:312321.

14. Ueda K, Hayashi M, Tanaka T, Hamano K. Omitting chest tube drainage after thoracoscopic major lung resection. EUR J CARDIO-THORAC. 2013; 44:225-229.

15. Liu CY, Hsu PK, Leong KI, Ting CK, Tsou MY. Is tubeless uniportal videoassisted thoracic surgery for pulmonary wedge resection a safe procedure? Eur $\mathbf{J}$ Cardiothorac Surg. 2020 Aug 1;58(Supplement_1): i70-i76.

16. Li S, Jiang L, Ang KL, Chen H, Dong Q, Yang H, et al. New tubeless videoassisted thoracoscopic surgery for small pulmonary nodules. Eur J Cardiothorac Surg. 2017; 51:689-693.

17. Zhang J, Dong S, Chu X, Lin S, Yu R, Jiang B, et al. Randomized Trial of an Improved Drainage Strategy Versus Routine Chest Tube After Lung Wedge Resection. The Annals of Thoracic Surgery. 2020; 109:1040-1046.

18. Zhang JT, Tang YC, Lin JT, Dong S, Nie Q, Jiang BY, et al. Prophylactic airextraction strategy after thoracoscopic wedge resection. THORAC CANCER. 2018; 9:1406-1412. 
19. Hirai K, Usuda J. Uniportal video-assisted thoracic surgery reduced the occurrence of post-thoracotomy pain syndrome after lobectomy for lung cancer. J THORAC DIS. 2019; 11:3896-3902.

20. Bendixen M, Jorgensen OD, Kronborg C, Andersen C, Licht PB. Postoperative pain and quality of life after lobectomy via video-assisted thoracoscopic surgery or anterolateral thoracotomy for early stage lung cancer: a randomized controlled trial. LANCET ONCOL. 2016; 17:836-844.

21. N.M. Rahman, J. Pepperell, and S. Rehal, et al, Effect of Opioids vs NSAIDs and Larger vs Smaller Chest Tube Size on Pain Control and Pleurodesis Efficacy Among Patients With Malignant Pleural Effusion: The TIME1 Randomized Clinical Trial, JAMA. 314 (2015) 2641-2653. 


\section{Figure Legends}

Fig 1. Procedure showing the position of routine 20 -Fr chest tube and 7-Fr doublelumen central venous catheter after VAT lung wedge resection
(A) Insertion of the chest tube
(B) Insertion of the alternative thoracic drainage catheter

Fig 2. Consolidated Standards of Reporting Trials (CONSORT) flow diagram

$\mathrm{CT}$, routine chest tube group; VC, central venous catheter group 
Figures
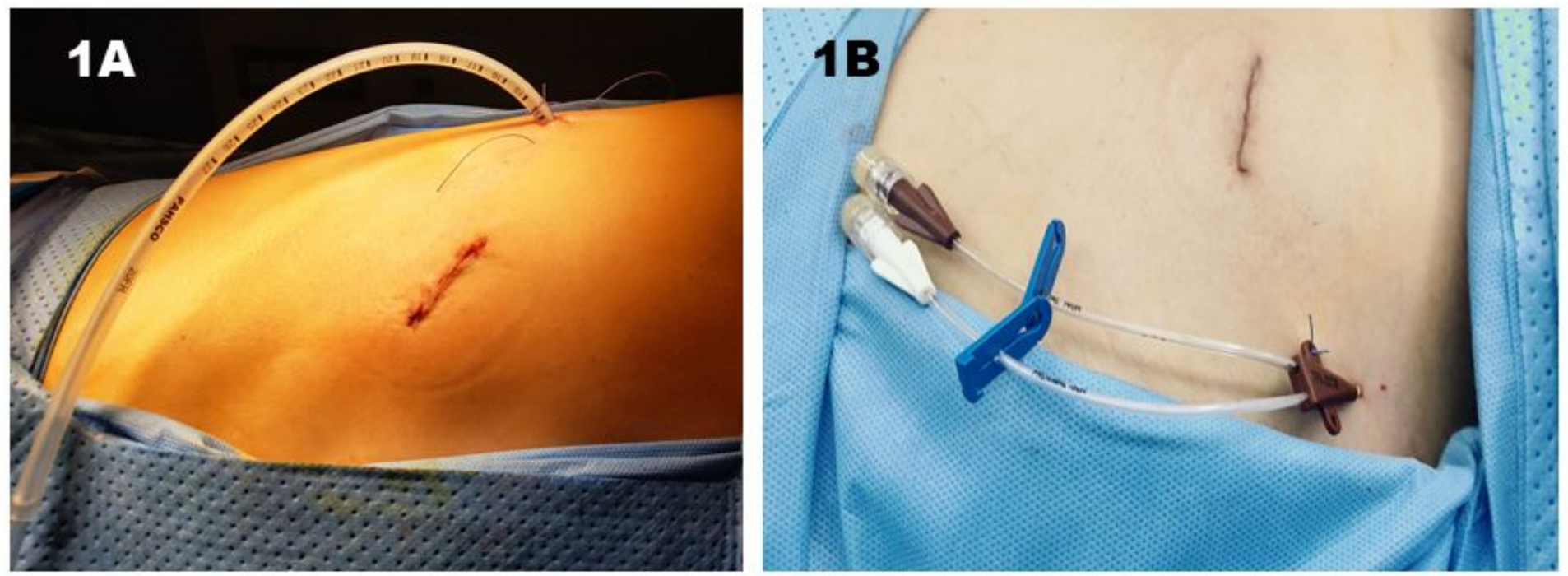

\section{Figure 1}

Procedure showing the position of routine 20-Fr chest tube and 7-Fr double-lumen central venous catheter after VAT lung wedge resection (A) Insertion of the chest tube (B) Insertion of the alternative thoracic drainage catheter 


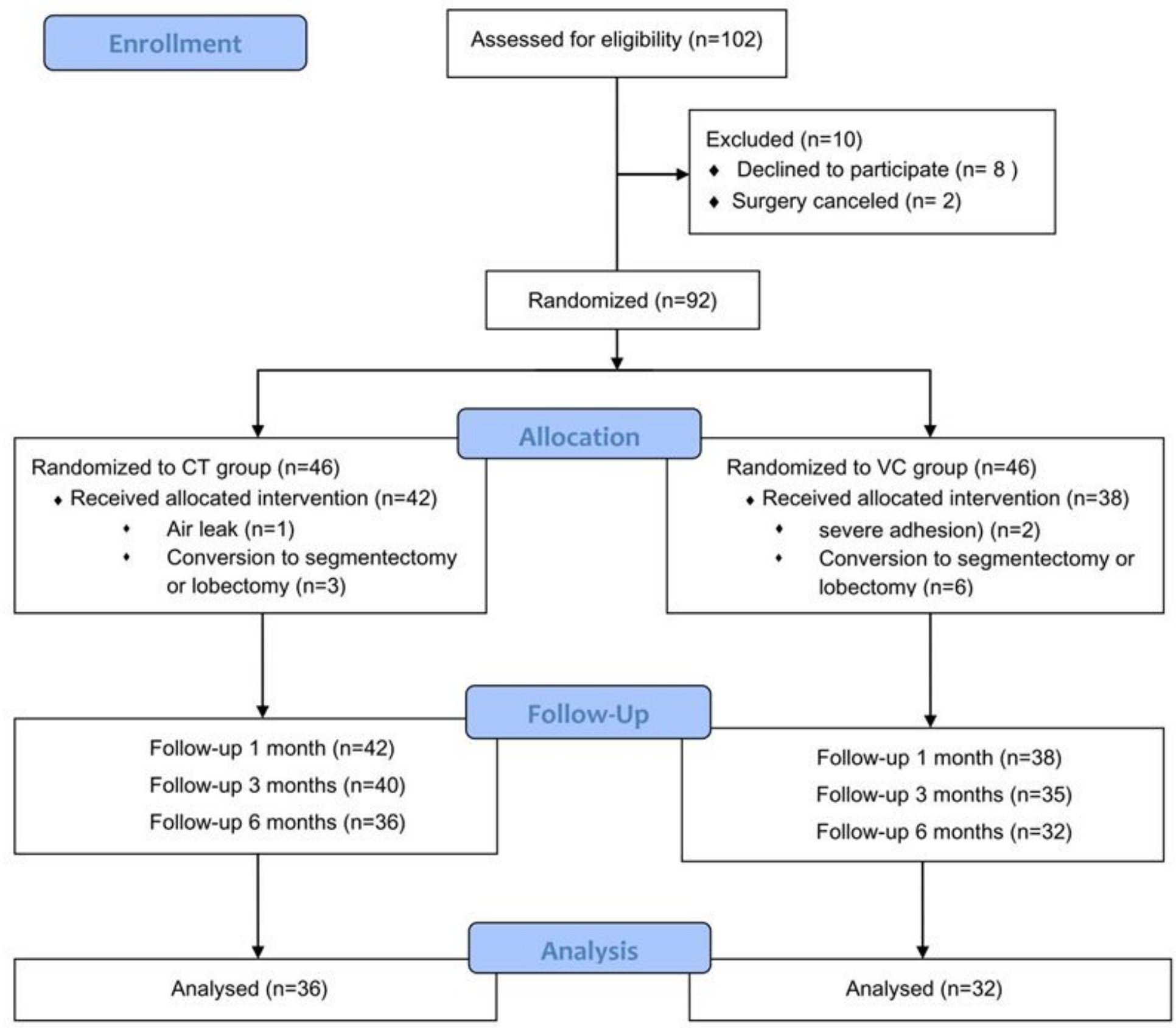

Figure 2

Consolidated Standards of Reporting Trials (CONSORT) flow diagram CT, routine chest tube group; VC, central venous catheter group 